\title{
Comunidad y contexto epistémico en la prueba pericial
}

\author{
Epistemic Context and Community in \\ Expert Evidence
}

Florencia Rimoldi*

Recepción: 4/10/2019

Evaluación: 12/10/2019

Aceptación final: 12/11/2019

\begin{abstract}
Resumen: En este comentario deseo abordar el trabajo de Vázquez a partir de consideraciones epistemológicas que, según mi propuesta, son relevantes para comprender el contexto judicial de averiguación de hechos y sus exigencias. Utilizo estas consideraciones para iluminar algunos aspectos de la práctica que pueden tener impacto en la apreciación de la propuesta de la autora. Para esto, este comentario se divide en tres partes. En la primera, ofrezco una síntesis crítica de la propuesta de Vázquez. En la segunda, argumento que algunas discusiones provenientes de la epistemología deberían incorporarse al modelo epistemológico ofrecido por la autora para dar una imagen más completa de las diferentes interacciones epistémicas que tienen
\end{abstract}

Doctora en Filosofía. Instituto de Investigaciones filosóficas, UNAM, Ciudad de México, México. Correo electrónico: florencia.r@filosoficas.unam.mx. Programa de Becas Posdoctorales en la UNAM, Becaria del Instituto de Investigaciones Filosóficas, asesorada por el doctor Miguel Ángel Fernández Vargas. Me gustaría expresar aquí mi agradecimiento a la revista Discusiones, especialmente a Valeria Trotti y a Hernán Bouvier, por convocarme a comentar este artículo de Vázquez. Como epistemóloga, he aprendido muchísimo de su trabajo, $y$ he disfrutado enormemente pensar el tema de la prueba pericial a partir de mi área. En este sentido, cualquier error que haya podido cometer en la interpretación de las tesis de la autora es completa responsabilidad mía, pero si he realizado algún aporte significativo a sus propuestas es gracias a lo que he aprendido "argumentativo-deferencialmente" de la lectura de sus trabajos. 
lugar durante el proceso en cuestión. En la tercera retomo las propuestas más salientes de Vázquez relacionadas con estos puntos para considerarlas a la luz de los mismos.

Palabras clave: Testimonio experto, justificación epistémica, contexto epistémico, comunidad.

\begin{abstract}
In this set of comments I want to take issue with Vázquez's work from the point of view of epistemological considerations that, as I see them, are relevant for the proper understanding of the judicial context of fact-finding and its demands. I use these considerations to shed light over some aspects of such practice that may have an impact in the author's proposal. To do so this paper is divided in three sections. The first section presents a critical synthesis of the Vázquez's proposal. In the second section I argue that some discussions originated in epistemology should be included in the epistemological model offered by the author, in order to present a more complete image of the different epistemic interactions that take place during the process in question. In the third part I go back to consider the most salient proposals from Vázquez related to these issues in order to approach them from the point of view of these discussions.
\end{abstract}

Keywords: Expert testimony, epistemic justification, epistemic context, community.

En este comentario deseo abordar el trabajo de Vázquez a partir de consideraciones epistemológicas generales que, según mi propuesta, son relevantes para comprender el contexto judicial de averiguación de hechos y sus exigencias. El propósito del comentario es utilizar estas consideraciones para iluminar algunos aspectos de la práctica que pueden tener impacto en la apreciación de la propuesta de la autora. En algunos casos, reforzando sus conclusiones, y en otros motivando un alejamiento de algunas propuestas puntuales. Para esto, este comentario se divide en tres partes. En la primera ofrezco una breve síntesis crítica de la propuesta de Vázquez, ampliando algunos puntos epistemológicamente relevantes de la misma, como el concepto de comprensión. En la segunda parte, argumento que hay algunas discusiones provenientes de la epistemología que deberían incorporarse al modelo epistemológico 
ofrecido por la autora, para dar una imagen más completa de las diferentes interacciones epistémicas que tienen lugar durante el proceso en cuestión. Con ese propósito, primero abordaré la pregunta de si la epistemología del testimonio puede explicar exhaustivamente el vínculo epistémico entre jueces/zas y peritos/as, y argumentaré que una imagen más completa de dicho intercambio surge si pensamos en un modelo que llamo "argumentativo-deferencial". Luego, desarrollaré brevemente los insights de un tipo especial de contextualismo en relación con la justificación epistémica, y argumentaré que el contexto en el que se da el intercambio epistémico entre jueces/zas y peritos/as debe ser reconocido como un genuino contexto epistémico cuyas reglas y estándares de justificación no pueden extrapolarse del contexto científico. En la tercera parte, retomaré las propuestas más salientes de Vázquez relacionadas con estos puntos para considerarlas a la luz de los mismos.

\section{Las propuestas de Vázquez}

El artículo de Vázquez se enmarca en una línea de trabajo que la autora viene desarrollando sistemáticamente en distintos lugares (Vázquez, 2015, $2016,2018)$ y que da lugar a una posición compleja y comprehensiva de las múltiples facetas del uso de testimonio experto en el ámbito judicial. La posición es compleja y comprehensiva porque, siendo sensible a las particularidades de cada etapa procesal, de cada operador jurídico involucrado, con sus distintos posibles roles dentro del proceso judicial y dentro de cada etapa, y a las exigencias y problemas epistemológicos de cada una (aplicando con rigor insights centrales de la epistemología del testimonio y la filosofía de la ciencia), ofrece a la vez la posibilidad de conectar todas estas complejas facetas en una visión global del uso del testimonio experto en el contexto de la práctica judicial. Esta conexión está dada por el énfasis permanente de Vázquez en comprender cómo y en qué sentido cada parte de su análisis se conecta con las otras para dar lugar a una concepción general de la prueba pericial, asociada a una práctica judicial con las características epistémicas que queremos - o deberíamos querer - que esta tenga.

En este contexto más general, este trabajo se centra en ofrecer herramientas procesales vinculadas al tratamiento de la prueba pericial en las 
etapas de admisión y, sobre todo, a su práctica, que generen un contexto epistémicamente favorable para la última etapa de valoración. Fundamentalmente, sus propuestas y argumentos se centran en la consecución de una meta epistémica específica y, según ella, necesaria para que el juez o la jueza pueda decidir racionalmente en la valoración: la comprensión del razonamiento inferencial utilizado por los expertos y las expertas. La diferencia, señalada por la autora, entre meramente poseer una información determinada (por ejemplo, sobre cada una de las premisas del razonamiento probatorio) y comprender a dicho razonamiento es de crucial importancia si lo que buscamos es que el juez o la jueza sea capaz de tomar una decisión racional respecto del caso teniendo en cuenta la totalidad de la evidencia.

Me gustaría ampliar esta última observación, para comprender cabalmente su importancia. Comprender es un fenómeno epistémico que no se agota en la mera posesión (incluso justificada) de cierta información (aunque esta sea verdadera). Una persona puede aceptar con justificación una serie de proposiciones (por ejemplo, porque una autoridad epistémica competente se las facilitó testimonialmente) y no ser capaz de conectarlas entre sí de manera de captar cómo están vinculadas (lógica o epistémicamente), ni cómo esa relación permitiría comprender un fenómeno determinado. Una persona puede saber, gracias a la palabra de su médico o médica, que tiene una determinada patología $X$, que esa patología incluye los síntomas $a, b$ y $c$, y que en parte está relacionada con las características fisiológicas $d$ y $e, y$ ambientales $f$ y $g$. Sin embargo, puede no comprender por qué tiene $X$, y tampoco comprender a $X$ como fenómeno médico (qué es $X$, cómo se relacionan los síntomas $a, b$ y $c$, y las características $d, e, f \mathrm{y}$ $g$ entre sí para dar lugar a $X$, etc.). La epistemología de la comprensión nos permitirá sistematizar un poco esta idea. Para Kvanvig (2003), por ejemplo, comprender un fenómeno redunda en captar ciertas relaciones explicativas y de coherencia entre sus partes. Según Riggs (2003, p. 20), "requiere de una apreciación profunda [...] de cómo sus partes encajan entre sí, qué rol juega cada una en el contexto del todo, y el rol que juega en el esquema más grande de las cosas". Grimm (2011) intenta clarificar esta idea de captación o apreciación de la relación entre partes, enfatizando que se trata 
de una habilidad para (1) entender el impacto que ciertos cambios de las partes podrían, o no, tener en el sistema entero, y (2) para hacer inferencias correctas sobre los modos en los que las diferencias en los valores de verdad de las proposiciones involucradas influirían en las inferencias que se dan en el mundo actual. Estas habilidades, entonces, requieren de un pensamiento contrafáctico (qué pasaría si las cosas fueran diferentes) en relación con las partes del fenómeno a comprender y con el resto de las cosas con las que dicho fenómeno está conectado.

La comprensión, entonces, es un estado epistémico mucho más sofisticado que el mero conocimiento proposicional, y para el caso que nos compete, que es el razonamiento inferencial pericial, queda claro que comprender un razonamiento incluye una capacidad que se encuentra por encima y por debajo de la mera constatación de las premisas, y de la pretensión de que de las premisas se siga una cierta conclusión relevante para el caso. En este sentido, el juez o la jueza debería ser capaz de relacionar las premisas entre sí, de manera de comprender por qué se sigue de ellas la conclusión, con qué fuerza, cuál es el grado de probabilidad de cada premisa, y cómo se relaciona el razonamiento y sus partes con el resto de la evidencia, con la cuestión de si la parte demandada es culpable y con la decisión judicial que debe tomarse. Esto último requiere de la habilidad contrafáctica mencionada al final del párrafo anterior, toda vez que el juez o la jueza no solo debe considerar los márgenes de error o la probabilidad de cada pedazo de evidencia, sino que, en relación con estos, debe considerar cómo impactaría en la decisión final la posibilidad (contemplada en el concepto de margen de error) de que la evidencia sea falsa después de todo. Todas estas consideraciones forman una parte central de la valoración de la evidencia y es por ello que resulta crucial el señalamiento de la autora en relación con la necesidad de buscar la comprensión del razonamiento inferencial pericial para una correcta valoración de la prueba pericial, pues esas capacidades contrafácticas son precisamente las involucradas en esta etapa. Sin esa comprensión, no se podrá valorar adecuadamente la prueba, y por ende no se podrá tomar una decisión racional respecto del caso.

Un último punto importante en relación con la definición de la meta epistémica perseguida es una suerte de cualificación relacionada con la comprensión buscada. La comprensión es un fenómeno que viene en gra- 
dos (ver Gordon, 2017), y en ningún caso sería deseable que la comprensión buscada por el juez o la jueza equivalga a la que puede tener el experto o la experta. Como afirma la autora, esto exigiría del juez o la jueza que sean capaces de producir la prueba, lo cual es no solo innecesario sino sencillamente impracticable, pues se convertirían en el experto o la experta. Podemos decir, entonces, que la comprensión buscada en este caso debe ser la suficiente para valorar la prueba adecuadamente. Volveré sobre este punto en la segunda parte de este trabajo.

Luego de argumentar que no hay razones serias para pensar que los jueces o las juezas sean incapaces de comprender el razonamiento inferencial (lo cual justificaría la actitud deferencialista que se suele tener hacia los peritos o las peritas, pero a la vez, y por lo dicho en los párrafos anteriores, tornaría a la valoración misma de la prueba en una etapa que está en flagrante contradicción con la etapa de la práctica, toda vez que en la práctica se estaría asumiendo la falta de una capacidad que es prácticamente definitoria de la etapa de valoración), la autora pasa a ofrecer una serie de herramientas procesales que deberían estar disponibles en las etapas de práctica y admisión -o que, si ya lo están, deberían estar mejor gestionadas- para favorecer la consecución de la comprensión suficiente para valorar la prueba adecuadamente.

Es importante señalar que todas las propuestas de la autora se dan en un marco de consideraciones epistemológicas de diverso tipo, que no solo facilitan la comprensión de los intercambios entre los/as distintos/as agentes involucrados/as (por ejemplo tomando las conclusiones más relevantes de la epistemología del testimonio y del desacuerdo entre pares epistémicos) sino que determinan cuáles son las exigencias que es razonable tener (por ejemplo en la fundamentación de los informes periciales) y cuáles no (por ejemplo la demostración de cientificidad o la exigencia de certezas).

Las propuestas, entonces, se dividen en dos grupos. Aquellas que se ofrecen en la etapa de la práctica de la prueba, de las cuales me interesa señalar aquellas dos que más apuntan a fortalecer el intercambio epistémico entre jueces/zas y peritos/as: (1) permitir preguntas aclaratorias por parte del juez o la jueza durante el contradictorio, y (2) gestionar eventuales desacuerdos entre peritos/as de manera que se contemplen las distintas causas posibles del mismo. La primera propuesta es importantísima porque 
siguiendo, en concordancia con la fundamentación de sus propuestas, un modelo según el cual el/la juez/a es un/a agente epistémico/a autónomo/a que busca la comprensión, restringir sus intervenciones o intentar regular el contradictorio en relación con ellas, resulta absolutamente inapropiado y pernicioso desde el punto de vista epistémico. La segunda propuesta contempla varias alternativas, de las cuales me interesa recalcar la de contemplar la posibilidad de realizar juntas entre peritos/as y jueces/zas para que los/as primeros/as puedan considerar juntos/as cuáles son exactamente los puntos en los que desacuerdan, y que quienes juzgan puedan en función de esto retomar algunas preguntas y cuestiones aclaratorias.

Las propuestas que, por su parte, se ofrecen en la etapa del pre-trial, tienen que ver fundamentalmente con dos cuestiones. La primera se conecta con una necesidad de contar cuanto antes con la información completa del caso, para que peritos/as y juez/a puedan contar con que comparten la misma información, y estén de acuerdo sobre cuál es el objeto del peritaje. Esto evitaría la generación de desacuerdos no-genuinos entre peritos/as. Para esto, se sugiere que como mínimo haya un cierto control judicial sobre la información brindada a los/as peritos/as, y como máximo una audiencia entre partes, peritos/as y juez/a para tratar estos puntos. Creo que esta sugerencia es más importante que lo que la misma autora reconoce, porque es otro punto nodal de intercambio epistémico entre peritos/as y juez/a que, como veremos en las siguientes secciones, resulta crucial para generar el contexto epistémico adecuado. La segunda propuesta tiene que ver con admitir solo aquellas pruebas periciales que argumenten adecuadamente a favor de su fiabilidad. Esta propuesta se sigue naturalmente de la idea de que el/la juez/a no solo puede, sino que debe comprender el razonamiento inferencial para tomar una decisión racional. Sin embargo, Vázquez parece sugerir (en la introducción) que los criterios indirectos para evaluar la confiabilidad (criterios asociados al/a la perito/a, o al tipo de conocimiento en el que se basa) no serían tan necesarios ni eficaces. Cuestionaré en la tercera sección este aspecto negativo de su propuesta.

En la siguiente sección realizaré algunas consideraciones epistemológicas que sentarán las bases para revisitar, en la tercera sección, las propuestas de Vázquez. 


\section{Consideraciones epistemológicas}

En esta sección deseo introducir más elementos epistemológicos al análisis de la prueba pericial que, si bien complejizan aún más el modelo propuesto por la autora, creo que permiten apreciar con mayor claridad la importancia de incentivar todas aquellas propuestas que vuelvan más robusto el contexto epistémico compartido por los/as distintos/as operadores/as y en especial por peritos/as y jueces/as, y por otro lado parecerían demandar evaluaciones del/la perito/a que vayan más allá de la fiabilidad de la prueba que este presenta. Estos puntos los retomaré en la siguiente sección. Ahora me centraré en discutir dos cuestiones epistemológicas y su relevancia para la cuestión de la prueba pericial. Discutiré: (2.1) el modelo de intercambio epistemológico que resulta adecuado para comprender la dinámica entre el/la juez/a y el/la perito/a; y (2.2) los aportes contextualistas y pragmatistas a la cuestión de la justificación epistémica.

\subsection{Hacia un modelo de argumentación deferencial crítica}

$\mathrm{Al}$ inicio de la sección 3 de su trabajo, la autora señala la relevancia de comprender adecuadamente la dinámica epistémica que tiene lugar en la interacción entre el/la juez/a y el/la perito/a antes de embarcarse en la empresa de mejorar normativamente la práctica que tiene lugar en el proceso judicial de averiguación de hechos. Para esto, propone analizar el intercambio entre el/la juez/a y perito/a en términos de la epistemología del testimonio, teniendo en cuenta el hecho fundamental de que "La prueba pericial como fuente de conocimiento es un testimonio, es decir, obtenemos la información relevante a partir de un tercero" (sección 3).

La autora, en este sentido, se apoya en la teoría del testimonio de Lackey (2008) para explicar las distintas facetas involucradas en el testimonio pericial, que, lejos de constituir una mera transferencia de conocimientos de una parte a la otra, requiere de la participación crítica y autónoma de dos agentes epistémicos/as. Esto significa que quien recibe el testimonio, en este caso el juez o la jueza, no puede deferir ciegamente ante lo que le dicen, sino que debe evaluar críticamente el contenido del testimonio, y tener 
razones positivas para aceptarlo, para que el resultado del intercambio testimonial realmente constituya conocimiento o una creencia justificada. ${ }^{2}$

Para comprender mejor lo que quiero decir a continuación, haré una distinción entre el fenómeno epistémico del testimonio y el testimonio pericial como un fenómeno en principio diferente del anterior. ${ }^{3}$ Lo que haré, entonces, es dar sentido a esto último mostrando que si bien el testimonio pericial tiene un elemento testimonial importante, contiene un elemento argumentativo igualmente relevante que debemos considerar. Para esto, es importante volver para atrás y recordar que la meta epistémica del juez en relación con la prueba pericial es la comprensión del razonamiento inferencial. En este sentido (para tomar el mismo ejemplo que la autora) si la conclusión del razonamiento es $p$ : "el acusado mintió", se espera que el juez o la jueza comprenda suficientemente las razones que sostienen esa conclusión, y que surgen de la comprensión general que tiene del razonamiento pericial involucrado ofrecido por el perito o la perita.

Contrastemos esta necesidad con el fenómeno epistémico del testimonio, que puede describirse como una de las formas básicas por las cuales circula la información entre las personas:

Un/a hablante $H$ le dice a un/a oyente $O$ que p, en virtud de lo cual $O$ pasa a creer que $p$.

La característica distintiva del testimonio, aquello que lo diferencia de otros intercambios epistémicos entre personas, es precisamente el hecho de que pasamos a formar creencias a partir de la palabra de otras personas, y no, por ejemplo, a partir de los argumentos que nos ofrecen, o a partir de estrategias de disuasión. La diferencia entre el testimonio y los otros

2 Por una cuestión de simplicidad, dejo de lado aquí la discusión respecto de cuál es el estado epistémico relevante para el juez o la jueza. Para un desarrollo más profuso de esta discusión, ver Vázquez (2015, cap. 1). Como hemos argumentado (Rimoldi y Bouvier, 2017) parece que la creencia no es una actitud ni necesaria ni suficiente para que el juez o la jueza pueda tomar decisiones racionales.

3 No utilizo aquí la distinción propuesta por Coady (1992) entre testimonio natural y testimonio formal porque creo que este último es más amplio que el pericial, toda vez que incluye también al testimonio lego. 
dos casos, es que la persona afirma "p" y es en virtud de ese acto que pasamos a creer que p. ${ }^{4}$ En los otros dos casos, se ofrecen razones o motivos (diferentes de $\mathrm{p}$ ) que nos inducen a aceptar $\mathrm{p}$ (ya sea racionalmente, como es el caso de la argumentación, o por otros medios, como es el caso de la disuasión). Incluso cuando una persona nos ofrece un argumento y afirma p como conclusión del mismo, esa afirmación no constituye un testimonio puesto que ni se afirma $\mathrm{p}$ con la intención de dar a conocer $\mathrm{p}$ a partir de dicha afirmación (sino que se espera que se acepte $\mathrm{p}$ a partir de las razones esgrimidas), ni tampoco se pasa a creer que $\mathrm{p}$ en virtud de que $\mathrm{H}$ lo dijo (sino que se pasa a creer que $\mathrm{p}$ en virtud de las razones esgrimidas). ${ }^{5}$

$\mathrm{Si}$, como decíamos anteriormente, se espera que el juez o la jueza no solo obtenga el conocimiento (o la creencia justificada) de que p, sino que comprenda hasta cierto punto por qué p, mediante la comprensión del razonamiento inferencial involucrado, entonces el testimonio pericial no se agota ni consiste necesariamente en un intercambio testimonial en el sentido recién desarrollado, sino que incluye, y de manera importante, un intercambio de tipo argumentativo, el cual consiste en el ofrecimiento de razones positivas a favor de p, para mostrar o exhibir que p. ${ }^{6}$ El perito o la perita, la mayoría de las veces, debe compartir no solo su conclusión de que "el acusado mintió" sino las razones que apuntan directamente a esto, en la forma de un razonamiento inferencial.

La complejidad del caso del testimonio pericial, sin embargo, no se agota en el reconocimiento de la dimensión argumentativa del mismo porque, como bien señala la autora, la prueba pericial es una fuente de

4 Esto es una simplificación, el acto de habla puede no ser la afirmación, y la persona puede testificar que p a pesar de no decir explícitamente "p" de muchas maneras que no vienen al caso. Véase Lackey (2008, cap. 1); Welbourne (2001, caps. 5 y 6).

5 Estas consideraciones pueden verse con mayor exhaustividad (Welbourne, 2001, caps. 5 y 6).

6 Este aspecto argumentativo no puede ser absorbido ni siquiera por teorías del testimonio robustas como las de Lackey, que demandan razones positivas para aceptar el testimonio. Esto es así porque, para no perder la esencia del testimonio (i. e. pasar a creer que p en virtud de que alguien lo dijo) y hacer solapar su teoría con el fenómeno de la argumentación, Lackey exige razones positivas para aceptar el testimonio de que $p$, pero no razones directas a favor de $p$ (ver Lackey, 2008, pp. 181-183). Las primeras son solo razones indirectas a favor de $\mathrm{p}$, y consisten en información sobre la confiabilidad de la persona, su veracidad, etc. Volveré sobre esta diferencia en la sección 3 de este comentario. 
conocimiento para el juez o la jueza. Esto quiere decir que, en muchos casos (si no en la mayoría) el perito o la perita cumple una doble función difícil de conciliar: la de argumentar a favor de una cierta conclusión, y la de educar al juez o la jueza en cuestiones centrales para evaluar la solidez de los mismos argumentos que esgrime. Un ejemplo para entender esta complejidad puede surgir si pensamos en casos más sencillos. Cuando dos pares epistémicos (es decir, dos personas que comparten aproximadamente la misma evidencia, las mismas competencias o expertise respecto de la cuestión a discutir) se encuentran en un intercambio argumentativo, las premisas del razonamiento que lleva a concluir que $\mathrm{p}$ son conocidas por ambos de manera independiente. Esto quiere decir que el par que recibe el argumento solo debe evaluar la validez o calidad del mismo para aceptar p. Las premisas y su plausibilidad están dadas por sentado o representan el conocimiento común que ambos tienen. En el caso de la prueba pericial, sin embargo, el juez o la jueza no tiene conocimiento previo de muchas de las premisas centrales del razonamiento ofrecido (todas aquellas que surjan del conocimiento especializado que forma parte de la expertise del perito/a), ${ }^{7}$ sino que las recibe testimonialmente por parte del perito o la perito, ahora sí, en el sentido del intercambio testimonial epistémico descrito anteriormente. Esta situación de dependencia epistémica es ineludible, pues aun cuando el perito o la perita justifique mediante razones -es decir, mediante otros razonamientos inferenciales- la verdad de alguna de las premisas involucradas -por ejemplo la de ciertas generalizaciones independientes de los hechos del caso- en algún punto se detendrá (pues la cadena inferencial no puede ser infinita) y realizará alguna afirmación autoritativa ("este es el método más usado en el campo", "tres métodos diferentes, y respectivos experimentos replicados en cantidad de veces, apoyan tal generalización", "existe consenso en la necesidad de utilizar varias técnicas de diagnóstico para dar con un resultado fiable", "en los artículos $\mathrm{x}, \mathrm{y}, \mathrm{z}$, se exhibe la correlación entre los fenómenos a y b pero no

7 Es decir, todas aquellas que no representen los hechos del caso, cuestiones de sentido común, el conocimiento común que se presupone compartido, o hechos notorios, o conocimiento especializado adquirido por el juez o la jueza dada su experiencia con otros casos similares. 
se prueba la relación causal", etc.). ${ }^{8}$ Llegado este punto, el juez o la jueza no tiene otra opción que deferir. $Y$ es aquí donde se plantea una especie de enigma o situación dilemática para el juez o la jueza. Pues, respectivamente, puede evaluar de manera autónoma la calidad del razonamiento en términos de su validez (si asumo que las premisas son verdaderas, ¿ $m e$ comprometo a aceptar $\mathrm{p}, \mathrm{o}$ al menos que $\mathrm{p}$ es probable?), y puede tener razones independientes para aceptar algunas de las premisas (ya sea porque son los hechos del caso, o porque el perito o la perita le ha dado razones adicionales para aceptarlas), pero habrá algunas premisas o presupuestos del razonamiento cuya verdad la obtiene testimonialmente de la misma persona que le está brindado el argumento. Aquí, su capacidad general para evaluar razonamientos no le alcanza para tener completa autonomía sobre el razonamiento, toda vez que debe deferir tarde o temprano hacia el perito o la perita, y esta deferencia se transfiere de premisas a conclusión, casi de la misma manera en que lo hace la verdad. Si llamamos a esta dinámica “argumentación deferencial", cabe realizarnos la siguiente pregunta: ¿de qué forma puede un juez o jueza aceptar de manera epistémicamente virtuosa una conclusión p surgida de una argumentación deferencial?

Si no comprendemos bien cómo esta dinámica puede resultar en un intercambio epistémico virtuoso, entonces podríamos tener derecho a sospechar que la meta buscada, a saber, la comprensión suficiente para valorar la prueba adecuadamente, no es completamente asequible. Pero hay un modelo en el que una dinámica similar se da y, prima facie, nos parece un intercambio virtuoso. La "argumentación deferencial" ocurre cuando leemos un artículo académico orientado a personas ajenas al campo de investigación del que trata, en el que se brindan argumentos muy completos y detallados que somos capaces de evaluar, pero tarde o temprano nos topamos con afirmaciones que solo están sustentadas por notas al pie que contienen referencias que no es razonable que busquemos, luego de

8 Aun cuando dichas afirmaciones cuenten con las referencias apropiadas en el informe escrito, lo que estas referencias permiten es una posible constatación independiente de la verdad de lo dicho. Pero mientras que esta constatación no se realice (y entiendo que esto por default no se hace), desde el punto de vista epistemológico la aceptación de esas premisas será un caso de aceptación surgida de un intercambio testimonial. 
lo cual compartimos críticamente las conclusiones del autor o la autora. ${ }^{9}$ La tarea, entonces, es entender qué herramientas procesales permiten que el juez o la jueza se asemeje más a una persona competente que lee un artículo científico y comparte críticamente las conclusiones de los autores o las autoras, y menos a una persona competente que lee un artículo pseudocientífico - por ejemplo negando la injerencia del hombre en el cambio climático- y comparte críticamente las conclusiones de los autores o las autoras, lo cual no es epistémicamente virtuoso. Retomaré esta cuestión en la siguiente sección.

\subsection{La justificación epistémica: aportes contextualistas}

Existe un supuesto epistemológico universalista muy arraigado no solo en la tradición epistemológica analítica sino también en el "sentido común" de las personas, académicas o no, de que para cualquier conjunto evidencial E (es decir, cualquier conjunto compuesto por distintos trozos de evidencia), si no tuviéramos limitaciones cognitivas, ni físicas, ni de tiempo, sería verdadero que para toda proposición empírica $\mathrm{p}, \mathrm{E}$ apoya $\mathrm{p}$ en cierto grado de manera objetiva. Esto significa que, dado E, habría solo una actitud racional hacia p porque las reglas epistémicas (las que determinan cómo y de qué forma un tipo de evidencia apoya a una determinada conclusión) son universales. Aunque este supuesto ha sido cuestionado desde muchos lugares, el supuesto universalista se cuela, en versiones más o menos sofisticadas, en muchos de los presupuestos de nuestros razonamientos y en la autoconcepción de nuestras prácticas. Esto es especialmente cierto en el caso de algunos/as científicos/as.

De las críticas que existen al universalismo, me interesa señalar aquí una originada en una forma especial de contextualismo defendida por Williams (2007), a la que él llama "contextualismo wittgensteiniano", tomando varios insights de Wittgenstein en On Certainty (Wittgenstein, 1969). Según Williams, lo único que hay son prácticas justificatorias (i.e., prácticas de dar y pedir razones) que si bien tienen un aire de familia, no

9 Quizás este modelo sea aplicable a ciertos contextos formativos superiores, como cursos de doctorado. 
sería legítimo unificarlas en algo como una teoría general de la justificación, que ofreciera reglas generales para el buen razonamiento, o determinara de una vez y para siempre los principios epistémicos que dicen cuándo una proposición está apoyada por un conjunto evidencial determinado, y contra los cuales todas las prácticas locales podrían compararse, explicando hasta qué punto se acercan hacia ese ideal, y considerando los factores "no epistemológicos" que explican por qué se alejan de este (por ejemplo institucionales, de tiempo, éticos, de poder, etc.). Esto va más allá del reconocimiento de la existencia de metodologías diferentes según el campo disciplinar (que es compatible con el universalismo toda vez que este reconozca tipos de conocimiento), y apunta a cuestionar la relevancia $y$, sobre todo, la pertinencia de intentar asociar y extrapolar atribuciones de justificación de un contexto a otro, incluso en contextos disciplinares similares. La justificación de teorías, creencias, hipótesis o proposiciones depende siempre del contexto particular o disciplinar en el que se estén considerando, y en este sentido una teoría, hipótesis, etc., puede estar justificada para un cierto contexto, pero no estarlo para otro.

No es este el lugar para defender aquí esta posición, pero sí creo dos cosas. La primera es que definitivamente esta es la posición más razonable que se puede tener, si uno no desea postular normas epistémicas que floten en un magma de "realidades filosóficas" que están por encima y por debajo de los fenómenos empíricos que, por supuesto, incluyen nuestras distintas prácticas sociales e institucionales. ${ }^{10}$ La segunda es que, si una posición como esta es la correcta, entonces esto tiene consecuencias muy relevantes para la cuestión de los intercambios epistémicos entre el/la juez/a y el/la perito/a y su gestión. Para apreciar mejor esta relevancia, veamos con algo más de detalle cuáles son los elementos que permitirían determinar el contexto en virtud del cual se puede juzgar si una creencia está o no justificada.

En general, lo que determina en qué contexto justificatorio estamos es todo aquello que se presupone en dicho contexto y, por ende, no será cuestionado. Los presupuestos de un contexto pueden ser explícitos o estar escasamente articulados, e incluyen principios metodológicos o epistémicos, y

${ }^{10}$ Para ver argumentos a favor de una posición como esta (aunque no específicamente a favor de esta posición) consultar Rimoldi (en prensa). 
también proposiciones particulares que se dan por sentadas. En este sentido, Williams señala cuatro tipos de factores que moldean el contexto justificatorio en el que estamos y, por ende, determinan los presupuestos del mismo.

Factores semánticos. Determinan qué tipo de cuestionamientos resultan inteligibles para un contexto y cuáles no. El tipo de factores que pertenecen a este grupo es de lo más diverso. Puede incluir verdades matemáticas, pero también ciertos juicios perceptivos. Lo que distingue a estos factores es que, si se cuestionaran en el contexto determinado por ellos, sencillamente no sabríamos cómo interpretar ese cuestionamiento. Por poner solo un ejemplo para el contexto judicial, sería difícil entender qué estaría cuestionando exactamente una parte que, en mitad de un juicio, le preguntara al juez “Cómo sé que usted es realmente un juez?”.

Factores metodológicos o disciplinares. Determinan qué tipo de cuestionamientos resultan legítimos en función de la investigación que estamos llevando a cabo, y de cuál es la dirección de la misma. Los presupuestos en este caso constituyen aquellas cuestiones a partir de las cuales podemos avanzar en la búsqueda de conocimientos. En este sentido, y como observa Wittgenstein $(1969, \$ 163)$, no cuestionar la legitimidad general de las fuentes documentales es una necesidad metodológica de, por ejemplo, la investigación historiográfica sobre los últimos años de Napoleón. Y en el contexto judicial, no sería legítimo cuestionar en medio de un juicio la fiabilidad general de las cadenas de custodia. Porque esto impediría llevar adelante la investigación, por ejemplo, respecto de la culpabilidad o inocencia del demandado. Tal como afirma Williams (2007) "las necesidades metodológicas son presuposiciones que están en pie de tal modo que cuestionarlas nos llevaría a cuestionar la competencia entera de la forma de investigación que ellas permiten. En este sentido, determinan el meta-contexto disciplinar de tales prácticas...” (p. 102). ${ }^{11}$

${ }^{11}$ Es importante señalar que estos presupuestos no son "intocables", incluso dentro de un mismo contexto. Con el tiempo, los presupuestos pueden corroborarse empíricamente (si una investigación que presupone a p arroja resultados importantes, entonces p se considera indirectamente validada hasta cierto grado) y también cuestionarse y revisarse de manera individual. Lo que no puede hacerse sin cambiar de contexto es cuestionar todas las presuposiciones a la vez. 
Factores dialécticos. Determinan qué tipo de cuestionamientos son apropiados en función del estado actual de la discusión en el campo disciplinar, las técnicas disponibles, los hallazgos y problemas relevantes, etc. Es decir, son todas aquellas presuposiciones que no quedan excluidas del campo mismo de investigación, y que dado el estado actual de esta no se cuestionan, pero podrían cuestionarse más adelante. La ciencia está plagada de ejemplos de cambios en este sentido. Por poner solo uno, durante mucho tiempo no tuvo sentido cuestionar la idea de que las neuronas no se regeneran una vez que el organismo está completamente desarrollado, y esto fue un presupuesto de múltiples investigaciones hasta que en 1998 nuevas investigaciones dieron lugar al descubrimiento de la neurogénesis. En el contexto jurídico, podría estar sucediendo algo similar con el valor probatorio de las confesiones. Pero esto es una mera especulación mía.

Factores económicos o pragmáticos. Estos presupuestos determinan fundamentalmente (aunque no exclusivamente) los estándares requeridos para considerar una proposición suficientemente justificada, y por ende, qué desafíos relacionados con esto son legítimos y cuáles no. Una vez que admitimos la falibilidad de nuestros métodos, y nos damos cuenta de que la búsqueda de certeza está fuera de lugar, la pregunta por cuándo una proposición está suficientemente justificada, nos lleva a preguntarnos, “justificada, ¿para qué?". Y es en este sentido que las consideraciones pragmáticas determinan cuáles son los desafíos apropiados y cuáles no. Consideraciones de tiempo y de recursos entran dentro de estos factores. Pero quizás la consideración más importante de todas tiene que ver con cuáles son las consecuencias pragmáticas, éticas y morales de aceptar p, e, igualmente importante, de aceptar $\mathrm{p}$ y estar equivocado respecto de $\mathrm{p}$. Y aquí quizás sea conveniente tomar como ejemplo la misma proposición en dos contextos diferentes. Quizás un científico o una científica, en un procedimiento de rutina para saber qué sangre transfundir a un/a paciente, considere justificada la proposición "esta muestra de sangre es de tipo cero negativo" con la mera evidencia que le provee el haber realizado una sola vez el examen de tipificación ABO. Esto porque el examen es muy confiable y la posibilidad de error no tiene consecuencias mayores dado que cualquier persona puede recibir sangre tipo cero negativo. En el contexto jurídico, sin embargo, la misma evidencia podría ser insuficiente para afirmar con justificación 
que la muestra de sangre es de tipo cero negativo. Por ejemplo, porque de aceptar esa proposición, se condenaría al acusado por asesinato, y el riesgo de estar equivocado es mucho más alto dado que involucraría condenar a un inocente por un delito mayor ( $y$ dejar al verdadero asesino suelto).

Debería resultar claro, a partir de lo visto, que el contexto en el que el intercambio epistémico entre jueces/zas y peritos/as tiene lugar es un contexto epistémico genuinamente diferente que como tal debe ser analizado en sus particularidades. Este contexto tiene como meta fundamental la comprensión suficiente para valorar adecuadamente la prueba. De todos los factores analizados, dos parecen ser los más problemáticos en caso de no atender a las diferencias contextuales. El primero es el dialógico. El juez o jueza no solo no es un científico o científica, sino que, como vimos anteriormente, debe aprender del perito o perita y evaluar autónomamente lo que dice. Por esta razón, el tipo de desafíos que será legítimo realizar dependerá fundamentalmente del estado de la "investigación”, entre peritos/as y juez/a, respecto de la cuestión a analizar. El modelo de "argumentación deferida” propuesto anteriormente podría darnos alguna pauta sobre las formas posibles de estos desafíos.

El segundo factor relevante es el económico o pragmático. Si no se presta suficiente atención a las diferencias contextuales, un perito puede realizar evaluaciones de confiabilidad que no son apropiadas para el contexto, y esto puede llevar al juez o a la jueza a valorar inadecuadamente la prueba. Si no atendemos suficientemente al modo en que estas diferencias en el contexto pragmático producen un genuino contexto epistémico diferente, el juez o la jueza puede no ser capaz de valorar la prueba porque el desconocimiento del perito o la perita de cuáles son los estándares de justificación adecuados dado el contexto impactará en la confianza de sus afirmaciones y esta ignorancia se transferirá al juez o a la jueza. Es decir, el juez o la jueza se verá incapacitado/a de comprender suficientemente los hechos relevantes, para decidir en base a esos hechos. Un perito o una perita que no entiende las diferencias contextuales puede afirmar que un determinado examen tiene una probabilidad del 0.01 de margen de error. Sin embargo, la confianza en (i) la corrección de ese valor, y, sobre todo, en (ii) cómo impacta ese valor en la probabilidad de que este test en particular, realizado en esta muestra particular, tenga ese margen de error, y (iii) el 
significado exacto que tiene el resultado de este test - ¿nos permite identificar una persona en particular, o más bien un tipo de persona? - puede - o debería - verse severamente afectada por el contexto pragmático en el que se está. En la siguiente sección retomaré estas consideraciones.

En esta sección he argumentado que el intercambio epistémico entre el/la juez y el/la perito/a responde al modelo que he llamado "argumentativo deferencial" y que dicho intercambio genera un contexto epistémico genuinamente diferente del científico. Las preguntas que deben responderse son (i) ¿Cómo dar cuenta del modo en que dicho modelo permite un intercambio epistémico virtuoso que dé lugar a la comprensión buscada? (ii) ¿Qué consecuencias tiene en la práctica el reconocimiento de la existencia de un contexto epistémico genuinamente diferente? Responderé a estas preguntas en la siguiente sección, retomando las propuestas de Vázquez mencionadas en la primera sección.

\section{Las propuestas de Vázquez revisitadas}

En las últimas páginas de su artículo, Vázquez realiza dos observaciones altamente significativas.

La primera es que cualquier esfuerzo por mejorar las prácticas epistémicas nunca será suficiente si no se mejora la calidad del conocimiento científico-forense desde el principio: esto es, más inversión para realizar los estudios necesarios para evaluar apropiadamente la calidad y el alcance de los métodos utilizados, mayores controles, mayor estandarización, etc. Esto es sumamente importante e iluminador, porque pone el dedo sobre dos cuestiones que, creo, deben tenerse en cuenta por todos los actores involucrados en este proceso. La primera es que cualquier criterio que se proponga para mejorar las prácticas epistémicas de las distintas etapas de admisión, práctica y valoración de las pruebas, por más óptimo que sea, no está libre de error. En el caso del manejo de la prueba pericial, esto significa que el juez o la jueza puede hacer todo bien, desde el punto de vista epistémico, y aun así incorporar como evidencia una prueba pericial que tiene una conclusión falsa. Esto nos lleva al segundo punto, que es la falibilidad de la ciencia y de sus métodos. La ciencia, por más rigurosa 
que sea, tampoco está libre de error. Esto significa no solo que teorías y métodos que se consideran hoy como fuera de discusión el día de mañana pueden probarse falsas o inválidos, sino que además dichos métodos son falibles (tienen margen de error), y, quizás lo más importante de todo para el caso que nos compete, la evaluación de esos márgenes de error también es falible. Todo esto demanda no solo poner los mayores esfuerzos en mejorar la ciencia pericial, sino además tomar una decisión, a nivel legislativo, de cómo se va a distribuir ese margen de error.

La segunda observación refiere a la importancia de fortalecer los puentes entre la comunidad jurídica y la comunidad científica. Creo que las propuestas de Vázquez relacionadas con incentivar el intercambio epistémico entre juez/a y peritos/as, deben apreciarse en su importancia no solo en relación con la cuestión de fomentar la comprensión del juez o la jueza, sino además con fomentar la creación de espacios comunes entre jueces/as y peritos/as, en los que pueda producirse una genuina comunidad nueva que fortalezca la práctica que da lugar al contexto epistémico mencionado en la sección anterior. En este contexto, la asimetría de conocimientos entre juez/a y perito/a no es unidireccional, pues el perito o la perita debe aprender conocimientos y presupuestos relevantes de la práctica judicial, sin los cuales no puede ser un/a agente epistémico/a completamente funcional en este contexto, que no es el del laboratorio. En este sentido, no solo importa que el perito o la perita comprenda las partes relevantes del proceso judicial, sus fines, y las consecuencias del error, sino también que se cuente con información empírica sobre los tipos de errores más comunes presentes en las investigaciones periciales, incluidos los errores originados en excesos de confianza. Sería interesante invertir también en estudios y bases de datos que recopilen esta información.

Como he sugerido en la sección anterior, las reglas epistémicas surgen con posterioridad a la constitución de una práctica. No tiene sentido preguntarse a priori cuáles son las mejores reglas y los estándares adecuados. Esto nos hace pensar que cuanto más se estimule la creación de una comunidad epistémica perito-judicial, mayor será la calidad de cualquier criterio propuesto por el legislador atendiendo, por ejemplo, a las presuposiciones (semánticas, metodológicas, dialécticas y económicas) compartidas por dicha comunidad. Sin embargo, y habida cuenta de que los intercambios 
epistémicos entre jueces/as y peritos/as ya existen (de lo que se trata es de fortalecerlos), me permito cuestionar la sugerencia de la autora relacionada con prestar más atención a la fiabilidad de los métodos y técnicas, y menos a los criterios "indirectos" como la evaluación del/la perito/a y sus credenciales o la cientificidad del conocimiento que este/a detenta.

Si bien comparto con ella plenamente la necesidad de que el juez o la jueza comprenda suficientemente los razonamientos involucrados, y la necesidad de que se tenga razones de primera mano para juzgar la confiabilidad de los métodos y el conocimiento utilizado, creo que, si recuperamos el modelo argumentativo-deferencial que he examinado en la sección anterior, podemos apreciar que las limitaciones que este modelo impone al ejercicio de la autonomía solo pueden minimizarse si adoptamos criterios "indirectos" para evaluar la confiabilidad de aquellas afirmaciones del/la perito/a que el/la juez/a no tiene manera de juzgar por sí mismo.

En la sección anterior, me preguntaba cómo el intercambio entre el/la juez/a y peritos/as puede ser epistémicamente virtuoso, asemejándose más al del convencimiento razonado a partir de un artículo científico y menos al convencimiento razonado a partir de un artículo pseudocientífico. Como dije anteriormente, ningún criterio, ni ningún conjunto de criterios, puede ofrecernos garantías absolutas. Pero mientras que el criterio que apunta a juzgar la confiabilidad de los métodos y generalizaciones involucradas en el razonamiento pericial nos permite eliminar una posible fuente de error vinculada al elemento argumentativo de la prueba pericial, las limitaciones de autonomía que tiene el modelo argumentativo-deferencial requieren criterios indirectos que nos permitan aceptar de manera crítica toda aquella información que recibimos de manera testimonial, para la cual no tenemos razones de primera mano, sino solo la palabra del/la perito/a. Es aquí donde, para el caso de los artículos científicos, importa dónde está publicado el artículo, en qué revista, si la revista tiene arbitraje ciego, quién es el/la autor/a, cuáles son sus credenciales, qué otras publicaciones tiene, si es honesto/a, si es responsable desde el punto de vista epistémico, etc. Ninguno de estos factores por separado garantiza que el testimonio sea confiable, y un testimonio puede ser confiable a pesar de no cumplir alguno de estos criterios. Algunos criterios, sin embargo, vinculados al/la autor/a, parecen ser excluyentes. Por ejemplo si el/la autor/a es deshonesto/a o irres- 
ponsable epistémicamente, no deberíamos deferir ante él/ella en absoluto. Pero cuando estos criterios se cumplen y varios de los otros están presentes, entonces parece razonable deferir ante el/la autor/a en algunas cuestiones, y juzgar por nosotros/as mismos/as la calidad de sus argumentos. Si, luego de haber leído críticamente el artículo, acompañamos la conclusión del/a autor/a, creo que esta actitud es perfectamente razonable, y lo seguiría siendo a pesar de que luego la conclusión resultara falsa. Y esto es lo más que se puede pedir a un/a agente epistémico real, tener las creencias que, dado el contexto, es racional creer. ${ }^{12}$

Aunque no sería legítimo extrapolar estos mismos criterios para la evaluación y admisión de un/a perito/a, lo cierto es que, vistos a la luz del modelo argumentativo-deferencial, los criterios Daubert para la etapa de admisión no parecen para nada inapropiados. Sin embargo, creo que hay dos tipos de criterio que no deberían ser exclusivos de una sola etapa. En relación con el informe pericial, creo que una breve evaluación mínima de la calidad de los argumentos presentados podría tener lugar en la admisión, a modo de "filtro". Es decir, a la exigencia sugerida por Vázquez de que en el informe pericial la fiabilidad sea susceptible de conocerse (dejando la tarea de la evaluación de ese informe a una etapa posterior) podría sumarse una exigencia adicional de que la argumentación ofrecida sea sometida a un mínimo análisis preliminar tal que, si se encuentran errores o saltos argumentales evidentemente no justificados, el/la juez/a tenga la potestad de excluir la evidencia sin más.

De la misma manera, los criterios de honestidad y responsabilidad epistémica deberían estar vigentes en todas las etapas del proceso. La importancia de que un/a perito/a sea responsable en sus dichos y en la estimación de los márgenes de error es casi tan alta como la confiabilidad de los métodos utilizados. Una persona sensible al contexto, sensible a los argumentos de los otros, a la evidencia contraria a sus propuestas, abierta a la crítica y a

${ }^{12}$ Creo que la consecuencia más importante del así llamado "escándalo Sokal" es precisamente que nunca se puede ser demasiado cauto cuando nos encontramos con una argumentación con la "apariencia correcta". Si la revista Social Text hubiera tenido en ese entonces la política editorial de evaluación por pares, el artículo muy probablemente no se habría publicado. Pero otra consecuencia igual de importante es que todas las prácticas presuponen la buena fe de los participantes. Sin este presupuesto de base, ninguna práctica puede tener lugar. 
la evaluación de pares, es la única capaz de ofrecer el testimonio confiable que la corte precisa. En este sentido, Anderson (2011) afirma:

La responsabilidad epistémica es la rendición de cuentas [accountability] sensible a la comunidad de investigadores. Las propias afirmaciones son sospechosas si uno no se somete y rinde cuentas a las demandas de justificación hechas por la comunidad de investigadores. Insistir en hacer ciertas afirmaciones, mientras se ignora evidencia y argumentos contrarios ofrecidos con pericia relevante, es ser dogmático. Ofrecer dichas afirmaciones como cosas que otros deberían creer por el sólo hecho de que nosotros lo decimos, mientras rechazamos rendir cuentas al respecto, es ser arrogante. Los dogmáticos no son confiables porque hay razones para creer que ellos están usurpando títulos de autoridad epistémica (p. 5).

La responsabilidad epistémica es una característica que evita varios vicios epistémicos muy perniciosos como por ejemplo el exceso de confianza en las conclusiones a las que se llega. Así como la comunidad médica está poniendo esfuerzos en prevenir la enorme cantidad de errores de diagnóstico producidos por el exceso de confianza en los médicos o las médicas, la comunidad jurídica debería estar preocupada en prevenir algo similar en el caso de los peritos o las peritas. Cassam (2017), al respecto del caso médico, realiza, como Anderson, una asociación importante entre arrogancia y exceso de confianza. Quizás podamos sistematizar un poco estas ideas atendiendo al modo en que puede evaluarse la responsabilidad epistémica en las diferentes etapas procesales.

En la etapa de admisión, la responsabilidad epistémica de un perito o una perita podría rastrearse con datos como si la persona tiene suficientes publicaciones sujetas a revisión de pares; ${ }^{13}$ si no ha defendido teorías o realizado investigaciones en campos disciplinares ajenos al propio; o si no

${ }^{13}$ En este sentido, las publicaciones con revisión de pares cumplen un doble propósito: son índice - hasta cierto punto- de la confiabilidad de la teoría o investigación propuesta, y son índice de responsabilidad epistémica. 
se ha asociado públicamente con instituciones o personalidades de cuestionable reputación. ${ }^{14}$

En la etapa de la práctica, y nuevamente atendiendo a las propuestas de Vázquez en relación con incentivar el intercambio epistémico entre jueces/ as y peritos/as, el juez o la jueza debería estar atento a signos de irresponsabilidad epistémica, como la irracionalidad dialógica (tanto con él/ella como con otros/as peritos/as); la arrogancia; y afirmaciones de certeza sobre las conclusiones y sobre los márgenes de error. En caso de encontrar estos signos, el juez o la jueza debería desconfiar fuertemente de la prueba pericial. Esto no significa que la prueba sea falsa, significa que el juez o la jueza no tiene las herramientas necesarias para valorar adecuadamente la prueba. No tiene la comprensión necesaria para hacerlo, porque la fuente de la que necesariamente debe depender presenta señales de vicios epistémicos que afectan la calidad de resultado.

\section{Conclusiones}

En este comentario he intentado enfatizar la importancia de las propuestas de Vázquez, haciendo hincapié en la necesidad de fortalecer de todas las formas posibles a la comunidad epistémica entre jueces/as y peritos/as, sin lo cual no podremos conseguir un intercambio epistémico virtuoso entre dichos agentes. He aportado, en este sentido, algunas herramientas epistemológicas que podrían utilizarse para mejorar la comprensión de cómo tendría que darse dicho intercambio virtuoso. Siguiendo a la autora, esto no quiere decir que parte del trabajo por mejorar la calidad de estos intercambios no resida en realizar mayores inversiones en mejorar la calidad del conocimiento científico. No solo el que es utilizado para realizar las pruebas periciales, sino también aquel que puede ofrecernos insights relevantes sobre qué información es necesaria para calibrar adecuadamente los estimados de confianza apropiados para el contexto epistémico científico-judicial.

${ }^{14}$ Estos son los criterios propuestos por Anderson (2011) para el caso de la evaluación del testimonio experto por parte de legos. 


\section{Bibliografía}

Anderson, E. (2011). Democracy, Public Policy, and Lay Assessments of Scientific Testimony. Episteme: A Journal of Social Epistemology, 8(2), 144-164.

Cassam, Q. (2017). Diagnostic Error, Overconfidence and Self-knowledge. Palgrave Communications, 3, 17025, https://doi.org/10.1057/ palcomms.2017.25.

Coady, C. A. J. (1992). Testimony. A Philosophical Study. Oxford: Clarendon Press Oxford.

Gordon, E. (2017). Understanding in Epistemology. Internet Encyclopedia of Philosophy.

Grimm, S. (2011). Understanding. En S. Bernecker y D. Pritchard (eds.), The Routledge Companion to Epistemology (pp. 84-94). New York: Routledge.

Kvanvig, J. (2003). The Value of Knowledge and the Pursuit of Understanding. New York: Cambridge University Press.

Lackey, J. (2008). Learning from Words. Testimony as a Source of Knowledge. Reino Unido: Oxford University Press.

Riggs, W. (2003). Understanding Virtue and the Virtue of Understanding. En M. DePaul y L. Zagzebski (eds.), Intellectual Virtue: Perspectives from Ethics and Epistemology (pp. 203-226). Oxford: Oxford University Press. Rimoldi, F. (en prensa). Una defensa genealógica del no-purismo en la justificación epistémica. Tópicos, Revista de Filosofía, CDMX, México.

Rimoldi F. y Bouvier, H. (2017). Assessing Testimony and Other Evidential Sources in Law: An Epistemological Approach. En F. Poggi y A. Capone (eds.), Pragmatics and Law: Practical and Theoretical Perspectives (pp. 439-468). Dordretch: Springer.

Vázquez, C. (2018). La im/parcialidad pericial y otras cuestiones afines. Isonomía, 48, 69-107.

Vázquez, C. (2016). La prueba pericial en la experiencia estadounidense. El caso Daubert. Jueces para la democracia, 86, 92-112.

Vázquez, C. (2015). De la prueba científica a la prueba pericial. MadridBarcelona: Marcial Pons. 
Williams, M. (2007). Why (wittgenstenian) Contextualism is not Relativism. Episteme: A Journal of Social Epistemology, 4(1), 93-114.

Wittgenstein, L. (1969). On Certainty. D. Paul y G. E. M. Anscombe (trads.). New York: Harper.

Welbourne, M. (2001). Knowledge. UK: Acumen. 